\title{
Power Laws of Wealth, Market Order Volumes and Market Returns
}

\author{
Sorin Solomon \\ Racah Institute of Physics, Hebrew University of Jerusalem, Israel \\ Peter Richmond \\ Department of Physics, Trinity College Dublin, Ireland
}

\begin{abstract}
Using the Generalised Lotka Volterra (GLV) model adapted to deal with muti agent systems we can investigate economic systems from a general viewpoint and obtain generic features common to most economies. Assuming only weak generic assumptions on capital dynamics, we are able to obtain very specific predictions for the distribution of social wealth. First, we show that in a 'fair' market, the wealth distribution among individual investors fulfills a power law. We then argue that 'fair play' for capital and minimal socio-biological needs of the humans traps the economy within a power law wealth distribution with a particular Pareto exponent $\alpha \sim 3 / 2$. In particular we relate it to the average number of individuals $\mathrm{L}$ depending on the average wealth: $\alpha \sim L /(L-1)$. Then we connect it to certain power exponents characterising the stock markets. We obtain that the distribution of volumes of the individual (buy and sell) orders follows a power law with similar exponent $\beta \sim \alpha \sim 3 / 2$. Consequently, in a market where trades take place by matching pairs of such sell and buy orders, the corresponding exponent for the market returns is expected to be of order $\gamma \sim 2 \alpha \sim 3$. These results are consistent with recent experimental measurements of these power law exponents ([Maslov 2001] for $\beta$ and [Gopikrishnan et al. 1999] for $\gamma$ ).
\end{abstract}

Keywords: Pareto-Zipf, Lotka-Volterra, power law; random multiplicative process; wealth distribution; market returns.

\section{Power Laws and Logistic Equations}

It was first observed by Pareto [1897 sic!] that in an economy, the fraction $P(w)$ of people owning a wealth $w$ is proportional to a power of $w$ :

$$
P(w) \sim w^{-1-\alpha}
$$

For the last hundred years the value of $\alpha \sim 3 / 2$ changed little in time and across the various capitalist economies. 
Aoki and Yoshikawa [1999] quote Montroll [1978] to the effect that "almost all the social phenomena, except in their relatively brief abnormal times obey the logistic growth" [Lotka 1925, Volterra 1926]:

$$
d w / d t=A w-B w^{2}
$$

It was shown [Solomon and Levy 1996, Levy and Solomon 1996, Biham et. al 1998] that Generalized Lotka Volterra (GLV) systems explicitating the aggregated logistic equation (2) at the microscopic agents level lead to power law distributions of the form (1). One can therefore say that the careful reconsideration of the system (2), led to the solution of a 100 year old puzzle by a 75 year old equation.

In the next sections we

- introduce the GLV model,

- show that it reduces to a set of decoupled stationary linear stochastic equations.

- derive analytically the Pareto law for the relative wealth distribution in the GLV model.

- explain the Pareto exponent $\alpha \sim 3 / 2$ based on intrinsic biological and social constraints

- relate via the GLV dynamics the Pareto exponent $\alpha \sim 3 / 2$ to the exponents of various stock market distributions. In particular GLV suggests that distribution of the volumes of the individual buy/sell orders is a power law with an exponent $\beta \sim 3 / 2$. For the distribution of the trade-by-trade returns the predicted exponent is $\gamma \sim 3$.

\section{The Generalized Lotka - Volterra Model}

Let us consider the dynamical system:

$$
d w_{i}(t)=w_{i}(t+\tau)-w_{i}(t)=\left[\varepsilon_{i}(t) \boldsymbol{\sigma}_{i}+c_{i}\left(w_{1}, w_{2}, \ldots, w_{N}, t\right)\right] w_{i}(t)+a_{i} \Sigma_{j} b_{j} w_{j}(t)
$$

with $i, j=1,2, \ldots, N$.

The interpretation of the various terms is as follows:

- $d w_{i}(t)=w_{i}(t+\tau)-w_{i}(t)$ is the time evolution of the wealth of the individual $i$ during the time interval $\tau$.

- The random variables $\varepsilon_{i}(t)$ represent the purely stochastic fluctuations in the returns of the various investors and since we express the square standard deviations by the variables $\sigma_{i}^{2}$ one can assume without loss in generality that

$$
<\varepsilon_{i}(t)^{2}>=1
$$

- The systematic endogenous and exogenous trends in the returns are expressed by the arbitrary functions $c_{i}\left(w_{1}, w_{2}, \ldots, w_{N}, t\right)$.

- Since the average value, $m_{i}(t)=<\varepsilon_{i}(t)>$ can be absorbed in $c_{i}$, we may assume without loss in generality that:

$$
m_{i}(t)=<\varepsilon_{i}(t)>=0
$$

- the terms $a_{i} \Sigma_{j} b_{j} w_{j}(t)$ represent the wealth redistribution by taxes, salaries, subsidies, etc. More precisely $b_{j}$ represents how much of the wealth of the individual $j$ contributes 
to the total wealth to be redistributed while the coefficients $a_{i}>0$ represent the amount of wealth redistributed to the individual $i$.

Since an overall factor in the $b_{j}$ 's can be absorbed in an overall factor in the $a_{i}$ 's, one can assume without loss in generality that:

$$
\Sigma_{j} b_{j}=1
$$

We will also assume here that all $\sigma_{i}^{2}, c_{i}$ and $a_{i}$ are of order $\tau$ and all $b_{j}$ 's are positive of order $1 / N$. Situations in which the coefficients describe certain neighbourhood structures in the investor's space have been studied in [Shnerb et. al 2000, 2001].

For arbitrary, unequal $c_{i}$ factors, the dynamics of (3) will lead to systematically increasing inequalities in the relative wealths $x_{i}$. In particular, individuals $i$ with very negative $c_{i}$ will keep loosing wealth. If they do it indefinitely, they will eventually disappear from the market (see however Levy, Persky and Solomon 1996, Farmer 1999, Solomon and Levy 2000, for other periodic, quasi-periodic and more complex regimes). The disappearence of the weakest, together with a host of other phenomena (adaptability, exploitation until exhaustion of systematic gain opportunities, adverse market effects: the largest players bidding against oneselves) leads usually to the "efficient market" regime. In simple words, "market efficency" means that the market is more efficient than any one individual in discovering the 'correct' prices and eliminating thereby the possibility of systematic speculative gain. While it is interesting to study the effects of eventual departures from "market efficiency" and their duration [Farmer 1999, Levy, Perski and Solomon 1996, Solomon and Levy 2000] it is reasonable to assume that for long time intervals, market efficiency holds and that except for the purely stochastic factors $\varepsilon_{i}(t)$ all the individuals have the same expected relative returns. i.e:

$$
c_{i}\left(w_{1}, w_{2}, \ldots, w_{N}, t\right)=c\left(w_{1}, w_{2}, \ldots, w_{N}, t\right)
$$

Then, in the limit

$$
a_{i} / \boldsymbol{\sigma}_{i}^{2}>>1 / \ln N
$$

one can show [Biham et al 1998, Huang and Solomon 2000, 2001, Blank and Solomon 2000 ] that the global dynamics of the economy can be expressed by taking the sum of the equations (3) each weighted by $b_{j}$ to obtain:

$$
d u(t)=u(t+\tau)-u(t)=c\left(w_{1}, w_{2}, \ldots, w_{N}, t\right) u(t)+a u(t)
$$

where

$$
\begin{gathered}
a(t)=\Sigma_{i} b_{i} a_{i}(t) \\
u(t)=\Sigma_{j} b_{j} w_{j}(t)
\end{gathered}
$$

and we have used equation (5) to neglect, in the limit $N \rightarrow \infty$, the sum of the random terms. One can think of the variable $u$ as a weighted average wealth.

Obviously for arbitrary functions $c\left(w_{1}, w_{2}, \ldots, w_{N}, t\right)$, the time evolution of $u(t)$ and the functions $w_{i}(t)$ can be very eventfull [Levy, Persky and Solomon, 1996, Farmer 1999, 
Levy, Levy and Solomon 2000, Solomon and Levy 2000]. However we show below that the probability distribution of the relative wealth

$$
x_{i}(t)=w_{i}(t) / u(t)
$$

has sometimes a much simpler behavior. By applying the chain differentiaton rule to (12) and using both (3) and (7) one gets:

$$
\begin{aligned}
d x_{i}(t) & =d w_{i} / u-w_{i} / u^{2} d u \\
& =\left[\varepsilon_{i}(t) \boldsymbol{\sigma}_{i}+c\right] x_{i}(t)+a_{i}-x_{i}(t)[c+a] \\
& =\left(\varepsilon_{i}(t) \boldsymbol{\sigma}_{i}-a\right) x_{i}(t)+a_{i}
\end{aligned}
$$

I.e. the system of equations splits into a set of independent, linear, time independent stochastic equations:

$$
d x_{i}(t)=\varepsilon_{i} \boldsymbol{\sigma}_{i} x_{i}(t)-a x_{i}(t)+a_{i}
$$

Note that this holds even if $\sigma_{i}, D_{i}$ and $a_{i}$ are functions of the corresponding $x_{i}$. In fact one obtains [Richmond 2000, Richmond and Solomon 2000, Solomon and Richmond 2000]:

$$
P\left(x_{i}\right)=\frac{\exp \left\{2 \int\left[-a x_{i}(t)+a_{i}\right] /\left[\boldsymbol{\sigma}_{i} x_{i}\right]^{2} d x_{i}\right\}}{\left[\boldsymbol{\sigma}_{i} x_{i}\right]^{2}}
$$

In the particular case when $\sigma_{i}$ and $a_{i}$ are constant, one obtains:

$$
P\left(x_{i}\right) \sim x_{i}^{-1-\alpha_{i}} \exp \left[\frac{-2 a_{i}}{x_{i} \boldsymbol{\sigma}_{i}^{2}}\right]
$$

where

$$
\alpha_{i}=1+2 a / \boldsymbol{\sigma}_{i}^{2}
$$

Note that in this approach one can have subpopulations that differ in their regular additive incomes, $a_{i}$ and/or in their standard deviation for the multiplicative (speculative) returns, $\boldsymbol{\sigma}_{i}^{2}$. In particular if has, for example, a sub-population involved in a NASDAQ like system with large $\boldsymbol{\sigma}_{i}^{2}$ this population will possess an $\alpha_{i}$ much smaller than the rest of the population engaged in classical or 'old economy' investment behaviour. This in turn will imply the concentration of wealth in a few 'lucky' hands as shown in section 4 . This will lead to market fluctuations which are significantly enhanced. This simly reflects the fact that wealth changes that occur are fractions of wealth of very wealthy individuals. (Think for example of the change in the market index induced by Bill Gates losing the current court case.) Obviously, a certain dependence of $a_{i}$ or $\sigma_{i}^{2}$ on $x_{i}$ may modify the power law exponent when substituted in (17).

In the case where all the coefficients are $i$-independent, i.e. $\boldsymbol{\sigma}_{i}^{2}=\boldsymbol{\sigma}^{2}, a_{i}=a$ and $b_{i}=1 / N$ one gets:

$$
P(x) \sim x^{-1-\alpha} \exp \left[-2 a /\left(\boldsymbol{\sigma}^{2} x\right)\right]
$$

with

$$
\alpha=1+2 a / \boldsymbol{\sigma}^{2}
$$

[Kesten 1973, Solomon and Levy 1996, Sornette and Cont 1997, Solomon 1998, Marsili, Maslov and Zhang 1998, Bouchaud and Mezard 2000]. The distribution $P(x)$ has a peak 
at $x_{0}=1 /(1+D / a)$. Above $x_{0}$, the relative wealth distribution $P(x)$ behaves like a power law while below $x_{0}, P(x)$ vanishes very fast.

One can show that for finite $N$, the main corrections are:

1 ) a factor which vanishes at $x=N$. This is consistent with the fact that there cannot be an agent with wealth $w_{i}(t)$ larger than the total wealth $N w(t)$ :

$$
P(x)=x^{-1-\alpha} \exp [-2 a /(x D)] \exp [-2 a /(D(1-x / N))]
$$

2) a correction to $\alpha$ :

$$
\alpha=1+2[a / D-K] /[1+K]
$$

where

$$
K=N^{-2+2 / \alpha} \sim N^{-4 a / D /(1+2 a / D)}
$$

This implies $\alpha<1$ if $N<<\exp (D / a)$ i.e. the wealth gets concentrated in just a few hands [Malcai, Biham and Solomon 1999, Blank and Solomon 2000, Huang and Solomon 2001].

\section{Why has the Pareto exponent been constant for the last 100 troubled years}

Until now, we have explained the survival of the individual wealth power law (18), (20), (22) in the presence of large time variations in the total wealth. We now relate the constant value of $\alpha \sim 3 / 2$ measured over the last 100 years (and for all the major capitalist economies) [Pareto 1897, Zipf 1949, Levy and Solomon 1997] to the social and biological constraints imposed on any society. The main idea is to exploit the particular characteristics of the shape of the wealth distribution curve (20) in order to relate the power decay of the probability distribution at large wealth to the wealth distribution of the poorest agents. This is possible since both the exponent $\alpha=1+2 a / \boldsymbol{\sigma}^{2}$ of the power law for large wealth and the coefficient $2 a / \boldsymbol{\sigma}^{2}$ in the exponential of $-1 / \mathrm{x}$ that dominates low wealth behavior, depend on the single parameter $a / \boldsymbol{\sigma}^{2}$. Consequently, how poor the poor are allowed (or can afford) to be, determines the power low distribution of more wealthy agents via the ratio $a / \boldsymbol{\sigma}^{2}$. The crucial expression $a / \boldsymbol{\sigma}^{2}$ can be understood as the ratio between additive regular incomes (salaries, social security, services) and the purely random multiplicative incomes originating in speculative market activities.

One of the crucial characteristics of the distribution $P(x)(20)$ is the fact that as one goes to lower and lower $x \rightarrow 0$ values below it maximum $x_{0} \sim 1 /(1+D / a)$, its decay to 0 is extremely fast. In fact all its derivatives are 0 at $x=0$. Given this sharp decay at low $w$ values, one can assume that effectively, the distribution $P(x)$ vanishes below a certain minimal relative wealth $x_{m}$ (poverty bound). The value of $x_{m}$ can be estimated roughly by assuming that there are no individuals below it and that above it, the power law is fulfilled. Then one gets the relation between $\alpha$ and $\mathrm{x}_{m}$ from the identity

$$
\begin{gathered}
<x>=<x_{i}(t)> \\
=<w_{i}(t) / w(t)>=w / w=1
\end{gathered}
$$


which implies:

$$
\begin{aligned}
1 & =\left[\int_{x_{m}} x^{-\alpha} d x\right] /\left[\int_{x_{m}} x^{-1-\alpha} d x\right] \\
& =\left[-1 /(1-\alpha) x_{m}^{1-\alpha}\right] /\left[-1 /(-\alpha) x_{m}^{-\alpha}\right] \\
& =x_{m} \alpha /(\alpha-1)
\end{aligned}
$$

or:

$$
\alpha=1 /\left(1-x_{m}\right)
$$

[Ijiri and Simon 1977, Levy and Solomon 1996, Malcai et al 99, Blank and Solomon 2000]. According (21) this yields:

$$
x_{m}=1-1 / \alpha=1 /\left(1+1 / 2 \sigma^{2} / a\right)
$$

Based on (30), (31) one can now give a general scenario of how the internal interests and constraints within society lead to the actual value of $\alpha \sim 3 / 2$ measured repeatedly in various economies in the last 100 years. Suppose that in a given economy the wealth necessary to keep a person alive is $K$. Certainly, anybody having less than that will have a very destabilizing effect on the society, so the number of people with wealth less then $K$ should be negligible if that society is to survive. Let us now suppose that the average family supported by an average wealth, has in average $L$ members . Clearly they will need a wealth of order $K L$, otherwise the wage earners will seek to correct the situation by strikes, negotiations, elections or revolts. Note that in a sense, the wealth of the average family is the definition of the minimal amount for supporting $L$ dependents, since the prices of the prime necessities will always adjust to it (i.e. if the average wealth increases so will the prices of housing, services, etc.).

In short, while the poorest people (who may not be able to afford a family) will ensure for their own survival that they do not get less than $1 / L$ of the average, the average population will almost by definition take care that their income is at least $L$ times the minimal wealth. In this way they insure the survival of their offspring. All in all, we are lead to the conclusion that

$$
x_{m} \sim 1 / L
$$

is dictated by the compromise between the survival instincts of the individual and the reproduction interesses of the species. Using equation (30) one further predicts:

$$
\alpha=1 /\left(1-x_{m}\right) \sim L /(L-1) .
$$

These relations fit well the known numbers for typical capitalist economies in the last century: family size $L \sim 3-4$, poverty line (below which people get subsidized)

$$
x_{m} \sim 1 / 4-1 / 3
$$

and $\alpha \sim 1.33-1.5$.

Our other key conclusion is, therefore, that the lower bound on the relative poverty governs totally the overall relative wealth distribution. The details of the dynamics by which this distribution arises are of course complex and depend on the interactions in the system. 
Most likely, during periods of large speculative fluctuations $\sigma^{2}$ which can give rise to significant numbers of "nouveau riches" , regular wage earners will demand that the salaries, pensions and social security (all contributing to $a$ in) will increase in such a manner that the ratio $a / \boldsymbol{\sigma}^{2}$ returns to the value $1 / 6-1 / 4$ consistent with $x_{m}=1 /(1+$ $\left.1 / 2 \boldsymbol{\sigma}^{2} / a\right)=1 / L$ with $L \sim 3-4$. During 'bearish' periods (small $\sigma^{2}$ ) salaries $a$ will erode leading again to a ratio $a / \boldsymbol{\sigma}^{2}$ that is fixed around:

$$
a / \sigma^{2} \sim 1 /[2(L-1)] \sim 1 / 4-1 / 6
$$

. Consequently, the value of $\alpha \sim L /(L-1)$ is kept fixed too around $\alpha \sim 3 / 2-4 / 3$. The Pareto experimental result $\alpha \sim 1.4$ falls well inside this range.

One may object that at the begining of the century the number of children per couple was larger. However, one should keep in mind that at that time children started to earn their living at an early age, so the number of dependent children per wage earner was not far from 3-4. Moreover a slow shift towards larger values of $\alpha$ (1.5-1.7) (corresponding to lower $L$ values) seems to take place in modern economies with strong social security policies.

\section{Heavy Tails of Buy/ Sell Order Volumes and Mar- ket Returns Distributions in GLV}

We discuss here some practical implications for the finacial markets of the formal results obtained in the previous sections.

In particular we derive the values that GLV suggests for the exponents of the probability distributions of

- volumes of sell/buy orders $=$ the amounts that the traders request to buy or sell. GLV suggests an exponent of the order $\alpha \sim 3 / 2$.

- trade-by-trade returns $=$ the stock price variations from one trade to the next. GLV suggests and exponent $\gamma \sim 2 \alpha \sim 3$.

According to Eq. (3), the variation of an individual investment is to lowest order in $\tau$ the term proportional to $\sigma_{i} \sim \tau^{1 / 2}$ :

$$
d w_{i}(t)=w_{i}(t+\tau)-w_{i}(t)=\varepsilon_{i}(t) \boldsymbol{\sigma}_{i} w_{i}(t)
$$

That is, according the GLV model, the variations in the invested capital are proportional to the capital itself. The proportionality of individual investments and returns to the individual wealth is a crucial property of the capital and a crucial ingredient of the GLV (and LLS) model. It is consistent [Levy and Solomon 97] with the experimental fact that the distribution of annual individual returns fulfills a power law with an exponent $\alpha$ equal to the one of the wealth distribution $(3 / 2)$.

In fact, if one uses the experimental fact that the investors have constant relative risk aversion then, one can prove [Levy, Levy Solomon 2000] that their utility functions are powers of their wealth. This in turn implies that the fraction of wealth that such an 
investor considers as optimal to invest is a fraction (that depends on the investor's current expectations) of his personal wealth. This is to be contrasted with the implications of the non-realistic (experimentally rejected but often used for analytical expediency) constant absolute risk aversion assumption. This would lead to an exponential utility function which predicts personal investments which are independent on the investor's wealth.

It is therefore very reasonable to assume that each buy/ sell order volume is stochastically proportional to the wealth of the trader that emits it.

Therefore, a natural implication of the GLV model [Levy and Solomon 97, Solomon 2000 ] is that the volumes of the individual sell/buy orders are distributed too by a power law with an exponent $\beta \sim \alpha \sim 3 / 2$ equal to the exponent of the Pareto individual wealth distribution. This is confirmed by measurements reported by [Maslov 2001] and [Gopikrishnan et al. 2000] where the order volumes are distributed by a power law with exponent $\alpha<2$.

Let us now estimate the exponent of the distribution of market price variations from one trade to another (trade-by-trade returns). Since the value of a stock is proportional to the sum of the individual investments in that stock, the trade-by-trade returns are stochastically proportional to the volumes traded in the individual trades. In fact the stochastic correlations between trade volumes and absolute value of the returns are well documented in the literature. In an ideal market where trading and price fixing take place in a centralized way [LLS, Biham et al] via a market maker who matches globally all individual demands / ofers such as to saturate the overall market demand/ ofer, it follows that trade volumes (and trade-by-trade returns) obey power laws with the same exponents as the buy/ sell order volumes $\gamma \sim \beta \sim \alpha \sim 3 / 2$.

However, in most real markets, the trades take place by the matching of pairs of buy and sell orders with compatible prices. In such a case the volume of each trade is equal to the smallest among the volumes of the matched pair. The prediction for distribution of trade volumes is now very different: Since the probability for each of 2 matched orders to exceed (or equal) a certain volume $v$ is

$$
P(>v) \sim v^{-\alpha}
$$

the probability that both have a volume (equal or) larger than $v$ is the product:

$$
P(>v,>v) \sim P(>v) P(>v) \sim v^{-\alpha} v^{-\alpha} \sim v^{-2 \alpha}
$$

So the prediction of GLV for such a market measurement is that the trades volumes and the trade-by-trade returns will fulfill a power law with an exponent

$$
\gamma \sim 2 \alpha \sim 3
$$

While for very short times, and small returns the exponent $\gamma \sim 3$ in the returns power distribution is masked by various other effects (e.g. subtelties related to the differentiation between market orders and limit orders and the way one accounts for a large order that is saturated by the sequential matching with a series of smaller orders; this accounts also for some apparent discrepancies between [Maslov 2001] and [Gopikrishnan et. al 2000]), for a wide range of parameters, the empirical data confirm an exponent $\gamma \sim 3$ in the power tails of the market returns distributions [Gopikrishnan et al 1999]. 


\section{Conclusions}

By analyzing economic dynamics from a general viewpoint we have demonstrated features that are common to most economies. Assuming only weak generic assumptions on capital dynamics, we are able to obtain very specific predictions for the distribution of social wealth. A crucial assumption is that the capital market is fair, i.e. equal capitals have equal opportunities. Mathematically, this is expressed via the assumption that expectations for returns are independent of $i$. We show that in such a market, the wealth distribution among individual investors fulfills a power law. The balance between "fair play" for the capital and minimal socio-biological needs of the humans seems to trap the world economy into a power law wealth distribution which determines much of its dynamical and equilibrium properties and in particular the Pareto exponent $\alpha \sim 3 / 2$. Moreover, the model is consistent with the recent measurments of the power law exponents of the distributions of order volumes and market returns [Maslov 2001, Gopikrishnan et al. 1999].

This paper is dedicated respectfully to the memory of Herbert Simon.

\section{References}

P. W. Anderson in The Economy as an Evolving Complex System II (Redwood City, Calif.: Addison-Wesley, 1995), eds. W. B. Arthur, S. N. Durlauf, and D. A. Lane.

M. Aoki and H. Yoshikawa, Demand creation and economic growth, U. of Tokio, Ctr. for Int'l. Research on the Japanese Econ. 1999.

O. Biham, O. Malcai, M. Levy, S. Solomon, Phys. Rev. E 58, 1352 (1998)

A. Blank and S. Solomon "Power laws in cities population, financial markets and internet sites (scaling in systems with a variable number of components)"

Physica A 287 (1-2) (2000) pp. 279-288.

J. P. Bouchaud and M. M \'ezard, Physica A 282, 536 (2000)

Farmer J.D., "Market Force, Ecology and Evolution," e-print adap-org/9812005. See also J.D. Farmer and S. Joshi, "Market Evolution Toward Marginal Efficiency" SFI report 1999.

P. Gopikrishnan, V. Plerou, X. Gabaix, H. E. Stanley Statistical Properties of Share Volume Traded in Financial Markets; Phys. Rev. E. (Rapid Comm.), 62 (2000) R4493.

P. Gopikrishnan, V. Plerou., L.A.N. Amaral, M. Meyer and H.E. Stanley, Phys. Rev. E 60, 5305 (1999).

Z. F. Huang and S. Solomon, Power, Levy, Exponential and Gaussian Regimes in Autocatalytic Financial Systems, cond-mat/0008026, to appear in Eur. Phys. J. B and

"Finite market size as a source of extreme wealth inequality and market instability" to appear in Physica A.

Y. Ijiri and H. A. Simon, Skew Distributions and the Sizes of Business Firms (NorthHolland, Amsterdam, 1977).

H. Kesten, Acta Math. 131 (1973) 207.

M. Levy, S. Solomon Power Laws are Logarithmic Boltzmann Laws

International Journal of Modern Physics C , Vol. 7, No. 4 (1996) 595; adap-org/9607001 
M. Levy M, and S. Solomon (1997), Physica A 242, 90.

M. Levy, H. Levy and S. Solomon, "Microscopic Simulation of Financial Markets; from Investor Behavior to Market Phenomena" Academic Press, New York, 2000.

M. Levy, N. Persky, and S. Solomon, "The Complex Dynamics of a Simple Stock Market Model" International Journal of High Speed Computing, 8, 1996

A.J. Lotka, (editor) Elements of Physical Biology, Williams and Wilkins, Baltimore, 1925; O. Malcai, O. Biham and S. Solomon, Phys. Rev. E, 60, 1299, (1999).

R. Mantegna and H. E. Stanley, An Introduction to Econophysics: Correlations and Complexity in Finance Cambridge University Press, Cambridge, 1999.

M. Marsili, S. Maslov and Y-C. Zhang, Physica A 253,(1998) 403.

S Maslov, "Price fluctuations from the order book perspective - empirical facts and a simple model" Physica A, present issue. We thank the author for discussing with us the results prior to publication.

E. W. Montroll Social Dynamics and the Quantifying of Social Forces", Proc. Nat. Acad. Sci. USA, Vol 75 No 10, Oct, 1978.

V. Pareto, Cours d" economie politique. Reprinted as a volume of Oeuvres Compl'etes (Droz, Geneva, 18961965). V. Pareto, Cours d'Economique Politique (Macmillan, Paris, 1897), Vol. 2. V. Pareto, Le Cours d' ' Economie Politique (Macmillan, London, 1897).

S. Redner, Am. J. Phys. 58, 267 (1990) ; Eur. Phys. J. B4, 131 (1998).

P. Richmond, Power Law Distributions and Dynamic behaviour of Stock Markets, to appear in Eur. J. Phys 2001.

P. Richmond and S. Solomon, cond-mat/0010222, submitted to J. Quan. Finance.

Nadav M. Shnerb, Yoram Louzoun, Eldad Bettelheim, and Sorin Solomon, "The importance of being discrete: Life always wins on the surface", Proc. Natl. Acad. Sci. USA, Vol. 97, Issue 19, 10322-10324, September 12, 2000, http://xxx.lanl.gov/abs/adaprg/9912005]

N. M. Shnerb, E. Bettelheim, Y. Louzoun, O. Agam, S. Solomon, "Adaptation of Autocatalytic Fluctuations to Diffusive Noise", Phys Rev E Vol 63, No 2, 2001; http://xxx.lanl.gov/abs/condnat/0007097

S. Solomon and M. Levy, adap-org/9609002, Int. J. Mod. Phys. C7, (1996) 745

S. Solomon, in Decision Technologies for Computational Finance, edited by A.-P. Refenes, A. N. Burgess, and J. E. Moody (Kluwer Academic Publishers, 1998).

S. Solomon, Generalized Lotka-Volterra (GLV) Models and Generic Emergence of Scaling Laws in Stock Markets, in "Applications of Simulation to Social Sciences" „Eds: G Ballot and G. Weisbuch; Hermes Science Publications 2000.

S. Solomon and M. Levy, Market Ecology, Pareto Wealth Distribution and Leptokurtic Returns in Microscopic Simulation of the LLS Stock Market Model; http://arXiv.org/abs/condmat/0005416 ; To appear in the Proceedings of "Complex behavior in economics: Aix en Provence (Marseille), France, 2000".

S. Solomon and P. Richmond Stability of Pareto-Zipf Law in Non-Stationary Economies, http://xxx.lanl.gov/abs/cond-mat/0012479 to appear in the Proceedings of WEHIA 2000, Marseille.

D. Sornette and R. Cont ,in J. Phys. I France 7 (1997) 431

H.E. Stanley, L.A.N. Amaral, J.S. Andrade, S.V. Buldyrev, S. Havlin, H.A. Makse, C.K. Peng, B. Suki and G. Viswanathan, Scale-Invariant Correlations in the Biological and Social Sciences. Phil. Mag. B, vol. 77, 1998, p. 1373. 
V. Volterra [1926], Nature, 118, 558. 